\title{
Bayer Pattern Demosaicking Using Local-Correlation Approach
}

\author{
Rastislav Lukac, Konstantinos N. Plataniotis, and \\ Anastasios N. Venetsanopoulos
}

The Edward S. Rogers Sr. Dept. of Electrical and Computer Engineering, University of Toronto, 10 King's College Road, Toronto, M5S 3G4, Canada

\{lukacr, kostas, anv\}@dsp. utoronto.ca

\begin{abstract}
A new Bayer pattern demosaicking scheme for single-sensor digital cameras is introduced. The raw output from a sensor, mostly a charge coupled device (CCD) or a complementary metal oxide semiconductor (CMOS) sensor, with a Bayer filter represents a mosaic of red, green and blue pixels of different intensity. To interpolate the two missing color components in each spatial location and constitute the full color, camera output, the proposed method utilizes edge-sensing interpolation and correction steps. Since the correction step is suitable only for the image regions with high spectral correlation, otherwise is counter productive, the scheme is adaptively controlled through the comparisons between the correlation coefficient and the pre-determined parameter. The proposed method yields excellent performance, in terms of subjective and objective image quality measures, and outperforms previously developed CFA interpolation solutions.
\end{abstract}

\section{Introduction}

Single-sensor digital color cameras use color filter array (CFA) to separate incoming light into a specific spatial arrangement of the color components. Most popular solutions utilize a Red-Green-Blue (RGB) Bayer CFA pattern shown in Fig. 10 [2]. The raw output from a sensor, mostly a charge coupled device (CCD) or a complementary metal oxide semiconductor (CMOS) sensor, with a Bayer filter represents a mosaic of red, green and blue pixels of different intensity. Since the two color components are missing in each spatial location (Fig. 2), they must be interpolated from the spatially adjacent CFA data. The process of interpolating missing spectral components is known as CFA interpolation or demosaicking.

The proposed demosaicking method produces the full color, camera output using edge-sensing interpolation and correction steps. After initial interpolation of the $\mathrm{G}$ components, the method utilizes color-difference model of [1] to generate $\mathrm{R}$ and $\mathrm{B}$ estimates using both $\mathrm{R}$ and $\mathrm{G}$ or $\mathrm{B}$ and $\mathrm{G}$ components, respectively. Thus, the procedure produces more accurate outputs compared to the schemes operating on $\mathrm{R}$ or B components, only. In the next step, the color appearance of the restored image is improved, first through the correction of the interpolated $\mathrm{G}$ 


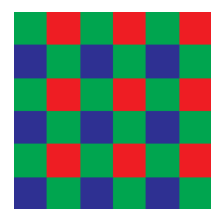

Fig. 1. RGB Bayer CFA pattern

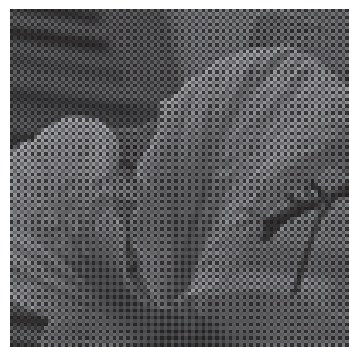

(a)

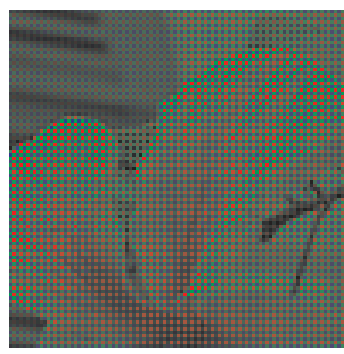

(b)

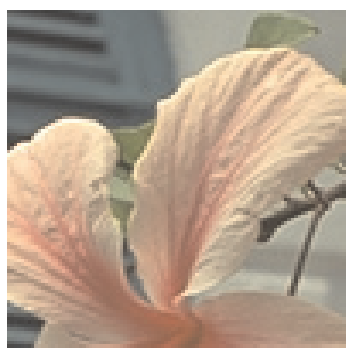

(c)

Fig. 2. Enlarged parts of the image Window arranged as: (a) a gray-scale Bayer image and (b) a color Bayer image, both acquired by a single-sensor digital camera, (c) a full color image captured by a three-sensor digital camera

components and then increasing precision of the previously interpolated $\mathrm{R}$ and $\mathrm{B}$ components. To restrict the correction process only for the image regions with high correlation among the spectral components, the local correlation characteristics are compared to the pre-determined parameter. This preserves image quality in the regions with weak correlation, where the correction step may be counter productive.

\section{Color Filter Array Basics}

Let us consider, a $K_{1} \times K_{2}$ gray-scale image $z(i): Z^{2} \rightarrow Z$ representing a twodimensional matrix of integer samples. In the Bayer CFA pattern, half of the pixels $z_{i} \in Z^{2}$, for $i=1,2, \ldots, K_{1} K_{2}$, correspond to the $\mathrm{G}$ channel, whereas $\mathrm{R}$, B channels are assigned the other half of the pixels. Assuming that $p=1,2, \ldots, K_{1}$ and $q=1,2, \ldots, K_{2}$ denote the spatial position of the pixels in vertical (image rows) and horizontal (image columns) directions, gray-scale pixels $z_{i}$ can be transformed into the RGB vectors $\mathbf{x}_{i}=\left(x_{i 1}, x_{i 2}, x_{i 3}\right) \in Z^{2}$, for $i=(p-1) K_{2}+q$, as follows:

$$
\mathbf{x}_{i}=\left\{\begin{array}{l}
\left(z_{i}, 0,0\right) \text { for } p \text { odd and } q \text { even } \\
\left(0,0, z_{i}\right) \text { for } p \text { even and } q \text { odd } \\
\left(0, z_{i}, 0\right) \text { otherwise }
\end{array}\right.
$$




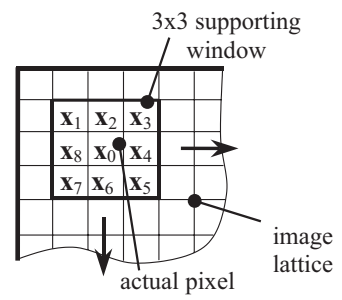

(a)

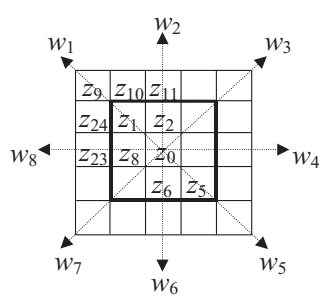

(b)

Fig. 3. Essential elements in the proposed scheme: (a) sliding supporting window determines the uncomplete color vectors $\mathbf{x}_{\mathbf{0}}, \mathbf{x}_{\mathbf{1}}, \ldots, \mathbf{x}_{\mathbf{N}-\mathbf{1}}$, (b) edge-sensing mechanism is expressed through the weighted coefficients $w_{1}, w_{2}, \ldots, w_{8}$

This transformation forms a $K_{1} \times K_{2}$ RGB image $\mathbf{x}(i): Z^{2} \rightarrow Z^{3}$ representing a two-dimensional matrix of three-component samples. Note that the color vectors $\mathbf{x}_{i}$ relate to one true component varying in $k$ from position to position, whereas other two components of $\mathbf{x}_{i}$ are set to zero. Estimating the missing color components of $\mathbf{x}(i)$ constitutes the interpolated RGB image $\mathbf{y}(i): Z^{2} \rightarrow Z^{3}$.

This process relates to a sliding supporting window $W=\left\{\mathbf{x}_{i} \in Z^{2} ; i=\right.$ $0,1, \ldots, N-1\}$ of finite size $N$, considered with the sample under consideration, sample $\mathbf{x}_{0}$, placed in the center of the window (Fig. 3 $\mathrm{a}$ ). The procedure replaces the center $\mathbf{x}_{0}$ by some function of the local neighborhood area $\left\{\mathbf{x}_{1}, \mathbf{x}_{2}, \ldots, \mathbf{x}_{N-1}\right\}$ at a time. The rationale of this approach is to minimize the local distortion and ensure the stationarity of the processes generating the image.

\section{Demosaicking Approach Based on the Local-Correlation Characteristics}

To follow structural information and interpolate missing image components in sharp shapes, efficient interpolation algorithms incorporate edge information into the interpolation process. The proposed method utilizes the edge-sensing mechanism of $[8]$ :

$$
\begin{gathered}
w_{1}=\frac{1}{1+\left(\left|z_{0}-z_{9}\right|+\left|z_{1}-z_{5}\right|\right) /(2 \sqrt{2})} \\
w_{2}=\frac{1}{1+\left(\left|z_{0}-z_{11}\right|+\left|z_{2}-z_{6}\right|\right) / 2}
\end{gathered}
$$

where $w_{1}$ and $w_{2}$ denote weights in north-west and north directions. The original gray-scale values $\left\{z_{0}, z_{1}, \ldots, z_{24}\right\} \in z(i)$ have an spatial arrangement shown in (Fig[3 $\mathrm{b})$. The weighting coefficients $w_{3}, w_{5}, w_{7}$ and $w_{4}, w_{6}, w_{8}$ are calculated applying appropriately the concept of (21) and (3), respectively.

Using eight weighting coefficients, the $\mathrm{G}$ channel is interpolated as follows:

$$
\bar{y}_{02}= \begin{cases}x_{02} & \text { if } z_{0} \cong x_{02} \\ \sum_{i=1}^{N-1} w_{i}^{\prime} x_{i 2}^{\prime} & \text { otherwise }\end{cases}
$$


where $N=9$ relates to a $3 \times 3$ sliding window, $z_{0}$ is the acquired gray-scale component positioned in the interpolated location, operator $\cong$ denotes a one to one relationship and $w_{i}^{\prime}=w_{i} / \sum_{j=1}^{N-1} w_{j}$ is the normalized weighting coefficient corresponding to pre-determined $\mathrm{G}$ values $x_{i 2}^{\prime}$. For illustration purposes, quantities $x_{(1) 2}^{\prime}$ and $x_{(2) 2}^{\prime}$ are given by:

$$
\begin{gathered}
x_{(1) 2}^{\prime}=\left(x_{(2) 2}+x_{(8) 2}\right) / 2+\left(\left(z_{1}-z_{5}\right) /(2 \sqrt{2})+\left(z_{11}-z_{0}+z_{23}-z_{0}\right) / 4\right) / 2 \\
x_{(2) 2}^{\prime}=x_{(2) 2}+\left(z_{11}-z_{0}+z_{2}-z_{6}\right) / 4
\end{gathered}
$$

Using the color-difference model of [1] and the $\mathrm{G}$ values obtained in (4), the $\mathrm{R}$ and $\mathrm{B}$ channels are estimated as follows:

$$
\bar{y}_{0 k}= \begin{cases}x_{0 k} & \text { if } z_{0} \cong x_{0 k} \\ \bar{y}_{02}+f_{(2 i) k}^{\prime \prime} & \text { if } z_{0} \cong x_{02} \\ \bar{y}_{02}+f_{(2 i-1) k}^{\prime \prime \prime} & \text { if } z_{0} \cong x_{0(k \pm 2)}\end{cases}
$$

where $k=1$ and $k=3$ characterize the $\mathrm{R}$ and $\mathrm{B}$ components, respectively, and the quantities $f_{(2 i) k}^{\prime \prime}$ and $f_{(2 i-1) k}^{\prime \prime \prime}$ are defined via

$$
\begin{gathered}
f_{(2 i) k}^{\prime \prime}=\sum_{i=1}^{(N-1) / 2} w_{i}^{\prime \prime}\left(x_{(2 i) k}-\bar{y}_{(2 i) 2}\right) \\
f_{(2 i-1) k}^{\prime \prime \prime}=\sum_{i=1}^{(N-1) / 2} w_{i}^{\prime \prime \prime}\left(x_{(2 i-1) k}-\bar{y}_{(2 i-1) 2}\right)
\end{gathered}
$$

with the normalized weights $w_{i}^{\prime \prime}=w_{2 i} \sum_{j=1}^{(N-1) / 2} w_{2 j}$ corresponding to edges in north, east, south and west directions. The weighting coefficients $w_{i}^{\prime \prime \prime}=$ $w_{(2 i-1)} / \sum_{j=1}^{(N-1) / 2} w_{(2 j-1)}$ correspond to diagonally positioned edges.

The utilization of the correction mechanism in the interpolation process improves contrast and accuracy of the initially interpolated G channel. Using the color-difference quantities similarly as in (7), the $\mathrm{G}$ values are corrected as follows:

$$
y_{02}= \begin{cases}\bar{y}_{0 k}+g_{(2 i) k}^{\prime \prime} & \text { if } z_{0} \cong x_{0 k} \\ \bar{y}_{02} & \text { otherwise }\end{cases}
$$

where $g_{(2 i) k}^{\prime \prime}=\sum_{i=1}^{(N-1) / 2} w_{i}^{\prime \prime}\left(\bar{y}_{(2 i) 2}-\bar{y}_{(2 i) k}\right)$ is defined using the weighting coefficients $w_{i}^{\prime \prime}$ of (7).

Considering the corrected $\mathrm{G}$ values of (10) the update of $\mathrm{R}$ and $\mathrm{B}$ components is completed using the proposed approach as follows:

$$
y_{0 k}= \begin{cases}y_{0 k} & \text { if } z_{0} \cong x_{0 k} \\ y_{02}+h_{(2 i) k}^{\prime \prime} & \text { if } z_{0} \cong x_{02} \\ y_{02}+h_{(2 i-1) k}^{\prime \prime \prime} & \text { if } z_{0} \cong x_{0(k \pm 2)}\end{cases}
$$

where

$$
h_{(2 i) k}^{\prime \prime}=\sum_{i=1}^{(N-1) / 2} w_{i}^{\prime \prime}\left(\bar{y}_{(2 i) k}-y_{(2 i) 2}\right)
$$




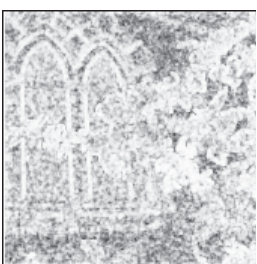

(a)

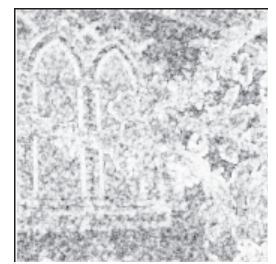

(b)

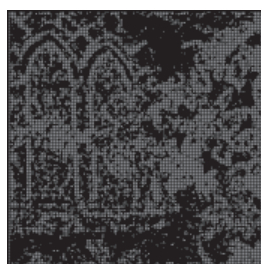

(c)

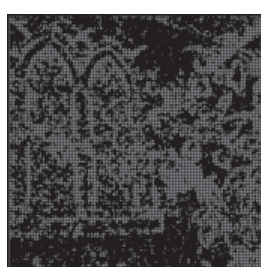

(d)

Fig. 4. Local correlation characteristics obtained using the image Window before (a,b) and after (c,d) thresholding: $(\mathrm{a}, \mathrm{c}) \mathrm{RG}$ correlation map and (b,d) GB correlation map

$$
h_{(2 i-1) k}^{\prime \prime \prime}=\sum_{i=1}^{(N-1) / 2} w_{i}^{\prime \prime \prime}\left(\bar{y}_{(2 i-1) k}-y_{(2 i-1) 2}\right)
$$

are defined using the weighting coefficients of (8)).

The correction procedure of (11) is performed only in image regions with the high spectral correlation. The method utilizes correlation characteristics (Fig 4) between the interpolated $\mathrm{G}$ channel of $\overline{\mathbf{y}}(i)$ and the original R,B channels of $\mathbf{x}(i)$. The correlation coefficient $C_{k}$ is defined over a $3 \times 3$ neighborhood as follows:

$$
C_{k}=\frac{\sum\left(x_{i k}-\hat{x}_{k}\right)\left(y_{i 2}-\hat{y}_{2}\right)}{\sqrt{\sum\left(x_{i k}-\hat{x}_{k}\right)^{2}} \sqrt{\sum\left(y_{i 2}-\hat{y}_{2}\right)^{2}}}
$$

where $i$, for $z_{i} \cong x_{i k}$, characterizes the spatial position of the samples corresponding to the original position of $\mathrm{R}, \mathrm{B}$ values in the Bayer pattern. The mean values $\hat{x}_{k}$ and $\hat{y}_{2}$ denote sample mean values related to the $k$-th original CFA components and the interpolated $\mathrm{G}$ components, respectively. Experimentation with a wide set of the test images showed that the correction operations should be performed in the image areas, where the local correlation coefficient is larger than $\beta=0.125$.

\section{Experimental Results}

A number of test color images have been used to evaluate the proposed demosaicking scheme. Examples are shown in Fig. 5. These images have been captured using professional three-sensor digital cameras. To facilitate the comparisons, the images have been normalized to a standard size of $512 \times 512$ pixels with a 8bits per channel RGB representation. Since the original Bayer image is usually unavailable in order to obtain test Bayer image data used in CFA interpolation researchers sample original images with the Bayer CFA pattern [10, 11.

The restored outputs are obtained using demosaicking (CFA interpolation) solutions. Results obtained via the proposed method are compared with those obtained using the bilinear interpolation (BI) scheme 10, the high definition color interpolation (HDCI) scheme [7], the median filtering (MFI) scheme [5], the effective color interpolation (ECI) approach [11, the alternative projection (AP) 


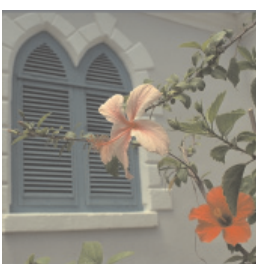

(a)

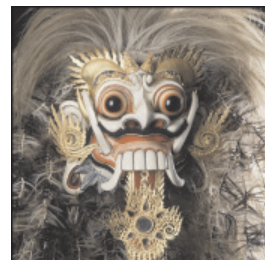

(b)

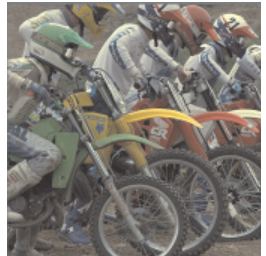

(c)

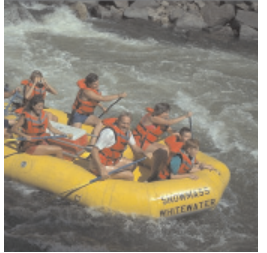

(d)

Fig. 5. Test color images: (a) Window, (b) Mask, (c) Bikes, (d) Rafting

Table 1. Obtained objective results

\begin{tabular}{crrrrrrrr}
\hline Image & \multicolumn{2}{c}{ Window } & \multicolumn{2}{c}{ Mask } & \multicolumn{2}{c}{ Bikes } & \multicolumn{2}{c}{ Rafting } \\
\hline Method & MSE & NCD & MSE & NCD & MSE & NCD & MSE & NCD \\
\hline BI & 35.1 & 0.0417 & 212.9 & 0.1328 & 157.1 & 0.1209 & 92.2 & 0.0753 \\
HDCI & 11.7 & 0.0252 & 94.8 & 0.0825 & 58.8 & 0.0744 & 45.3 & 0.0492 \\
MFI & 7.7 & 0.0239 & 57.3 & 0.0822 & 27.4 & 0.0620 & 25.0 & 0.0434 \\
ECI & 6.8 & 0.0228 & 49.2 & 0.0819 & 20.5 & 0.581 & 21.0 & 0.0396 \\
AP & 5.6 & 0.0212 & 42.3 & 0.0754 & 16.9 & 0.0534 & 20.6 & 0.0358 \\
C2D2 & 6.5 & 0.0193 & 56.3 & 0.0750 & 26.7 & 0.0545 & 22.5 & 0.0375 \\
SAIG & 11.5 & 0.0280 & 87.4 & 0.0854 & 53.1 & 0.0768 & 41.5 & 0.0507 \\
SHT & 21.3 & 0.0349 & 131.7 & 0.1070 & 94.8 & 0.1025 & 61.1 & 0.0626 \\
KA & 22.3 & 0.0324 & 60.2 & 0.0736 & 71.7 & 0.0792 & 55.7 & 0.0504 \\
Proposed & 4.4 & 0.0181 & 35.4 & 0.0648 & 12.1 & 0.0425 & 15.0 & 0.0308 \\
\hline
\end{tabular}

approach [6], the color correlation directional derivative (C2D2) scheme [8], the smooth hue transition approach (SHT) [4, the saturation based adaptive inverse gradient (SAIG) [3], and the Kimmel's algorithm (KA) 9]. The efficiency of the all these methods is measured, objectively, via the mean square error (MSE) and the normalized color difference criterion (NCD) [7].

Table 1 summarizes the results corresponding to restoration of the test images shown in Fig. 5. As it can be observed the conventional BI scheme introduces significant inaccuracy into the restoration process. Other techniques such as HDCI, MFI, ECI, AP, C2D2, SAIG and KA which utilize more advanced interpolators compared to the BI scheme, provide better results in terms of both objective criteria. It has to be mentioned that some sophisticated algorithms such as HDCI, SAIG, and KA often fail in image scenarios with color corresponding to zero or very small portion of any additive primary. In such a case, the aforementioned schemes produce color artifacts. 
(e)

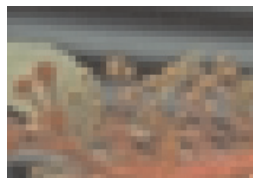

(f)

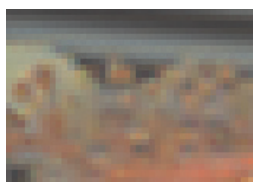

(g)

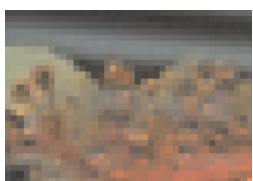

(h)

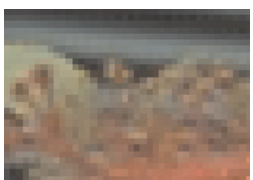

(i)

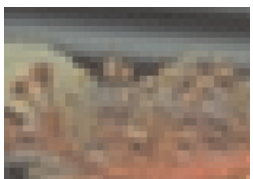

(j)

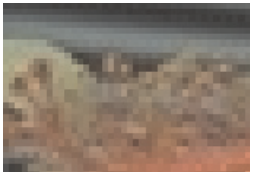

(k)

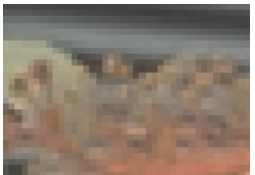

(1)

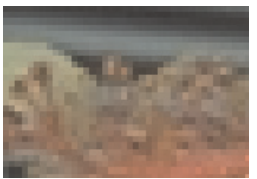

(a)
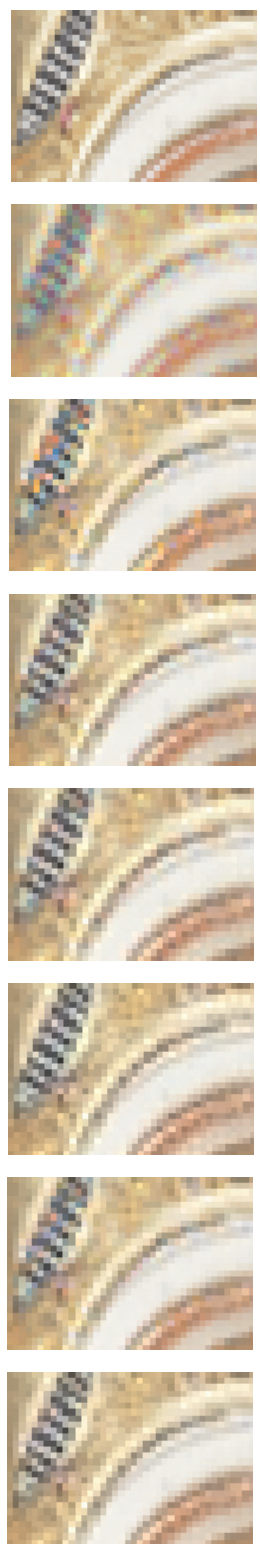

(b)
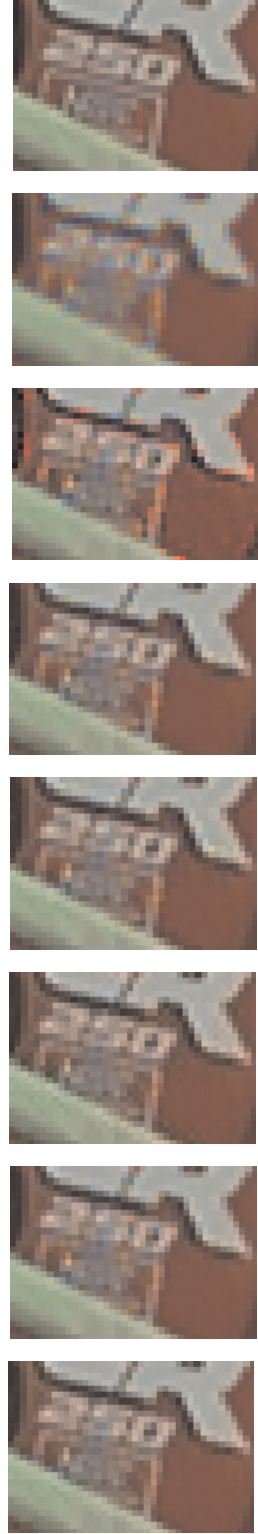

(c)
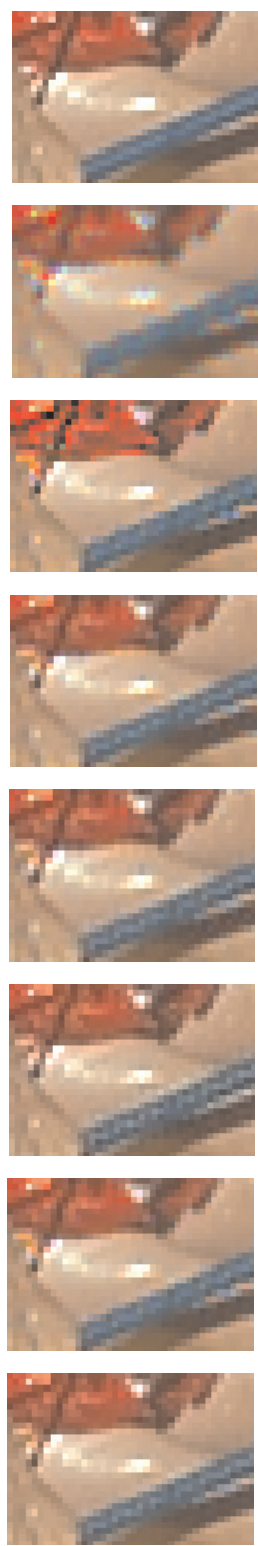

(d)

Fig. 6. Enlarged parts of the obtained results corresponding to the images: (a) Window, (b) Mask, (c) Bikes, (d) Rafting; and the methods: (e) original images, (f) BI output, (g) HDCI output, (h) MFI output, (i) ECI output, (j) AP output, (k) C2D2 output, (l) the proposed method output 
Figure 6 facilitates the visual comparison of zoomed parts corresponding to the original images as well as the restored outputs obtained using the methods under consideration. Results indicate that the BI and HDCI schemes blur image edges and delete both structural content and fine details ( Fig. 6f,g). The MFI, ECI, AP and C2D2 approaches exhibit improved detail-preserving characteristics ( Fig. 6h-k). It can be seen that the proposed method preserves the image details and avoids color artifacts ( Fig. 6). Excellent design characteristics of the proposed method allow to restore the image with the highest fidelity compared to the original. At the same time, the proposed solution preserves the original structural content. This results in visually pleasing, naturally colored outputs.

\section{Conclusion}

A new, edge-sensing CFA interpolation approach for single-sensor digital cameras was presented. The method utilizes local correlation characteristics and a color-difference model to produce full color camera output. Excellent design characteristics of the proposed method result in sharp, naturally colored interpolated images. At the same time, this produces significant improvements and excellent results compared to the previously developed CFA interpolation methods.

\section{References}

1. Adams, J.: Design of practical color filter array interpolation algorithms for digital cameras. Proc. of the SPIE, 3028 (1997) 117-125

2. Bayer, B.E.: Color imaging array. U.S. Patent 3971 065, (1976)

3. Cai, C., Yu, T.H., Mitra, S.K.: Saturation-based adaptive inverse gradient interpolation for Bayer pattern images. IEE Proceedings - Vision, Image, Signal Processing 148 (2001) 202-208

4. Cok, D.R.: Signal processing method and apparatus for producing interpolated chrominance values in a sampled color image signal. U.S. Patent 4642678 (1987)

5. Freeman, W.T.: Median filter for reconstructing missing color samples. U.S. Patent $5373322,(1988)$

6. Gunturk, B., Altunbasak, Y., Mersereau, R.: Color plane interpolation using alternating projections. IEEE Trans. Image Processing 11 (2002) 997-1013

7. Hur, B.S., Kang, M.G.: High definition color interpolation scheme for progressive scan CCD image sensor. IEEE Trans. Consumer Electronics 47 (2001) 179-186

8. Kehtarnavaz, N., Oh, H.J., Yoo, Y.: Color filter array interpolation using color correlation and directional derivatives. Journal of Electronic Imaging 12 (2003) 621-632

9. Kimmel, R.: Demosaicing: image reconstruction from color CCD samples. IEEE Trans. Image Processing 8 (1999) 1221-1228

10. Longere, P., Zhang, X., Delahunt, P.B., Brainard, D.H.: Perceptual assessment of demosaicing algorithm performance. Proceedings of the IEEE 90 (2002) 123-132

11. Pei, S.C., Tam, I.K., Effective color interpolation in CCD color filter arrays using signal correlation. IEEE Trans. Circuits and Systems for Video Technology 13 (2003) 503-513 